\title{
SUBNORMALITY AND QUASINORMALITY OF TOEPLITZ OPERATORS
}

\author{
TAKASHI ITO AND TIN KIN WONG
}

\begin{abstract}
Halmos asks whether every subnormal Toeplitz operator on $\mathrm{H}^{2}$ is either analytic or normal. It is shown that for a certain class of Toeplitz operators, the subnormality implies either analyticity or normality.
\end{abstract}

1. Introduction. Let $L^{2}$ be the Hilbert space of all square summable complex valued functions on the unit circle with respect to the normalized Lebesgue measure. The functions $e_{n}(z)=z^{n}, n=0, \pm 1, \pm 2, \cdots$, form the usual orthonormal basis of $L^{2}$. Let $H^{2}$ be the closed subspace of $L^{2}$ spanned by the basis functions $e_{n}$ for $n=0,1,2, \cdots$. Thus $H^{2}$ consists of all functions in $L^{2}$ whose Fourier coefficients vanish on all negative integers. A function is said to be analytic if it is in $\mathrm{H}^{2}$.

For a bounded measurable function $\varphi$ on the unit circle, the Toeplitz operator $T_{\varphi}$ is the bounded operator on $H^{2}$ which consists of multiplication by $\varphi$ followed by the orthogonal projection $P$ of $L^{2}$ onto $H^{2}$ :

$$
T_{\varphi} f=P(\varphi f), \quad f \in H^{2} .
$$

Among Toeplitz operators, we now consider two classes. One is the class of analytic Toeplitz operators, and the other is the class of all those Toeplitz operators which are normal. A Toeplitz operator $T_{\varphi}$ is said to be analytic if $\varphi$ is analytic. In this case, $T_{\varphi}$ turns out simply to be the multiplication operator induced by $\varphi$ on $H^{2}$. That is $T_{\varphi} f=\varphi f, f \in H^{2}$. The most fundamental analytic Toeplitz operator is the unilateral shift $U=T_{e_{1}}$ defined by the function $e_{1}(z)=z$. The class of normal Toeplitz operators has been characterized as those of the form $T_{\alpha+\beta \psi}$ where $\alpha$ and $\beta$ are complex numbers and $\psi$ is a bounded, real-valued, measurable function on the unit circle [2, Corollary to Theorem 9]. This is equivalent to saying that $T_{\varphi}$ is normal if and only if there is a complex number $\alpha$ with $|\alpha|=1$ such that $\hat{\varphi}(-n)=\alpha(\hat{\varphi}(n))$ for all $n \neq 0$, where $\hat{\varphi}(n)$ denotes the $n$th Fourier coefficients of $\varphi$.

Presented to the Society, January 22, 1971 ; received by the editors November 13, 1970. AMS 1970 subject classifications. Primary 47B20, 47B35; Secondary 46J15.

Key words and phrases. Toeplitz operator, subnormal operator, quasinormal operator, hyponormal operator.

(c) American Mathematical Society 1972 
These two classes of Toeplitz operators, the analytic ones and the normal ones, are essentially disjoint except for the scalar multiples of the identity. Every analytic Toeplitz operator is subnormal. (For an analytic Toeplitz operator $T_{\varphi}$, the multiplication operator induced by $\varphi$ on $L^{2}$ is a natural normal extension of $T_{\varphi}$ to $L^{2}$.) Thus the union of these two classes consists of Toeplitz operators which are subnormal. Moreover, among Toeplitz operators, analytic ones and normal ones are the only subnormal Toeplitz operators known to exist. In [3] Halmos posed the following question: Is every subnormal Toeplitz operator either analytic or normal? It is the purpose of this paper to show that for a certain class of $\varphi$, a subnormal Toeplitz operator $T_{\varphi}$ must be either analytic or normal. For instance, if $\varphi$ is a polynomial in an inner function ${ }^{1}$ $\chi$ and its complex conjugate $\bar{\chi}$, then subnormality of $T_{\varphi}$ implies either analyticity or normality of $T_{\varphi}$. (See Theorem 1.) In Halmos' question, even if the condition of subnormality is strengthened to quasinormality, the answer still seems to be unknown. However, we show that if $\varphi$ is either almost analytic or almost coanalytic, and $T_{\varphi}$ is quasinormal, then $T_{\varphi}$ is either normal or analytic (see Theorem 3 and its corollary).

If the condition of subnormality in Halmos' question is weakened to hyponormality, then the question can be answered in the negative. That is, there exist hyponormal Toeplitz operators which are neither analytic nor normal. An easy example is the Toeplitz operator $U^{*}+2 U$. This operator is also of interest because it is not a subnormal operator (see Theorem 1 or Remark 4), which leaves Halmos' question still open. (For another example of an operator which is hyponormal but not subnormal, see [4, Problem 160].)

2. Subnormal Toeplitz operators. For elementary properties of Toeplitz operators and subnormal operators we shall refer to BrownHalmos [2] and Halmos [4]. The following theorem gives some supporting evidence to an affirmative answer of Halmos' question.

THEOREM 1. Let $\varphi$ be a polynomial in an inner function $\chi$ and its complex conjugate $\bar{\chi}$. The Toeplitz operator $T_{\varphi}$ is subnormal if and only if it is either normal or analytic.

Proof. The sufficiency is clear. For the necessity, we first assume $\chi$ is the function $e_{1}(z)=z$. Suppose $\varphi$ is not analytic, and $T_{\varphi}$ is subnormal. Thus $\varphi=\hat{\varphi}\left(-n_{0}\right) e_{-n_{0}}+\cdots+\hat{\varphi}(0)+\cdots+\hat{\varphi}\left(m_{0}\right) e_{m_{0}}$ for some $n_{0}>0$ with $\hat{\varphi}\left(-n_{0}\right) \neq 0$. Notice that the self commutator $C_{\varphi}=T_{\varphi}^{*} T_{\varphi}-T_{\varphi} T_{\varphi}^{*}$ is of finite rank. To see this, let $k_{0}=\max \left\{n_{0}, m_{0}\right\}$. So $k_{0}$ is a positive integer. Clearly, $e_{k} \varphi$ and $e_{k} \bar{\varphi}$ are both analytic for all $k \geqq k_{0}$. Hence $\left\|T_{\varphi}^{*} e_{k}\right\|=\left\|T_{\varphi} e_{k}\right\|$ for all

\footnotetext{
1 An analytic function $\chi$ is said to be inner if $|\chi|=1$ a.e.
} 
$k \geqq k_{0}$. Consequently the kernel of $C_{\varphi}$ contains the closed subspace spanned by $\left\{e_{k} ; k \geqq k_{0}\right\}$. Therefore $C_{\varphi}$ has finite-dimensional range which is contained in the $k_{0}$-dimensional subspace $\left[e_{0}, e_{1}, \cdots, e_{k_{0}-1}\right]$.

Now let $N$ be a normal extension of $T_{\varphi}$ to a Hilbert space $K$. With no loss of generality, we may assume that $K=H^{2} \oplus H^{2}$. So $N$ has an operator matrix of the form

$$
\left(\begin{array}{cc}
T_{\varphi} & A \\
0 & B
\end{array}\right)
$$

where $A, B$ are operators on $H^{2}$ and 0 is the zero operator. By the normality of $N$, we have the following operator equations:

(i) $C_{\varphi}=A A^{*}$,

(ii) $T_{\varphi}^{*} A=A B^{*}$.

It follows from (i) that $C_{\varphi}$ and $A$ have the same range. Thus $A\left(H^{2}\right) \subset$ $\left[e_{0}, e_{1}, \cdots, e_{k_{0}-1}\right]$. Hence $A\left(H^{2}\right)$ consists of analytic polynomials of degree at most $k_{0}-1$.

We claim that $C_{\varphi}=0$. For if this were not so, we could choose some nonzero element $f$ in $C_{\varphi}\left(H^{2}\right)=A\left(H^{2}\right)$ of maximum degree, say $l$. Then $T_{\varphi}^{*} f$ would be an analytic polynomial whose degree is $n_{0}+l>l$ and with leading coefficient equal to $\left(\hat{\varphi}\left(-n_{0}\right)\right)-\hat{f}(l) \neq 0$. But from (ii), $T_{\varphi}^{*} f$ is in $A\left(H^{2}\right)$, and $T_{\varphi}^{*} f$ has degree $n_{0}+l>l$. This contradicts the choice of $l$. Hence $C_{\varphi}=0$, which means that $T_{\varphi}$ is normal.

Now suppose that $\varphi$ is a polynomial in an inner function $\chi$ and its complex conjugate $\bar{\chi}$, and that $T_{\varphi}$ is subnormal. Write $\varphi=\sum_{k=-n}^{n} \alpha_{k} \chi^{k}$ for some positive integer $n$. Then $T_{\varphi}=\sum_{k=0}^{n} \alpha_{k} T_{\chi}^{k}+\sum_{k=1}^{n} \alpha_{-k}\left(T_{\chi}^{*}\right)^{k}$. It is known that $T_{\chi}$ is a pure isometry with some multiplicity (finite or infinite). (See [7, Chapter 7].) So $T_{\chi}$ is unitarily equivalent to a direct sum of copies of the unilateral shift [5]. Namely $H^{2}$ is a direct sum of closed subspaces $N_{\lambda}, H^{2}=\sum_{\lambda} \oplus N_{\lambda}$, and each $N_{\lambda}$ is a reducing subspace of $T_{\chi}$. The restriction of $T_{\chi}$ to $N_{\lambda}, T_{\chi} \mid N_{\lambda}$, is unitarily equivalent to the unilateral shift $U$ on $H^{2}$. Let $V_{\lambda}$ be the unitary operator mapping $N_{\lambda}$ onto $H^{2}$ such that $V_{\lambda}\left(T_{\chi} \mid N_{\lambda}\right) V_{\lambda}^{*}=U$. Then we have

$$
V_{\lambda}\left(T_{\varphi} \mid N_{\lambda}\right) V_{\lambda}^{*}=\sum_{k=0}^{n} \alpha_{k} U^{k}+\sum_{k=1}^{n} \alpha_{-k}\left(U^{*}\right)^{k} .
$$

Since each $N_{\lambda}$ is a reducing subspace of $T_{\varphi}$, the operator $V_{\lambda}\left(T_{\varphi} \mid N_{\lambda}\right) V_{\lambda}^{*}$ is subnormal for each $\lambda$, and it is a Toeplitz operator on $H^{2}$ defined by the trigonometric polynomial $\sum_{k=-n}^{n} \alpha_{k} z^{k}$. Applying what we have established about trigonometric polynomials, we can conclude that either the trigonometric polynomial $\sum_{k=-n}^{n} \alpha_{k} z^{k}$ is analytic or $V_{\lambda}\left(T_{\varphi} \mid N_{\lambda}\right) V_{\lambda}^{*}$ is normal for all $\lambda$. If $\sum_{k=-n}^{n} \alpha_{k} z^{k}$ is analytic, then $\varphi=\sum_{k=-n}^{n} \alpha_{k} \chi^{k}$ is analytic. If $V_{\lambda}\left(T_{\varphi} \mid N_{\lambda}\right) V_{\lambda}^{*}$ is normal for all $\lambda$, then $T_{\varphi}$ is normal. This completes the proof. 
REMARK 1. Recall that a function $f$ in $L^{2}$ is called almost analytic if $e_{n_{0}} f$ is analytic for some positive integer $n_{0} . f$ is said to be almost coanalytic if its complex conjugate $\vec{f}$ is almost analytic. Let $\varphi$ be any bounded measurable function with Fourier expansion $\varphi=\sum_{k=-\infty}^{\infty} \hat{\varphi}(k) e_{k}$. Suppose that $T_{\varphi}$ is hyponormal. Then $\left\|T_{\varphi} e_{n}\right\| \geqq\left\|T_{\varphi}^{*} e_{n}\right\|$ for all $n=0,1,2, \cdots$. It follows that

$$
\sum_{k>n}|\hat{\varphi}(k)|^{2} \geqq \sum_{k<-n}|\hat{\varphi}(k)|^{2} \quad \text { for } n=0,1,2, \cdots .
$$

If $\varphi$ is almost coanalytic and $T_{\varphi}$ is hyponormal, then $\varphi$ must be a trigonometric polynomial. To see this, write $\varphi=\sum_{k=-\infty}^{\infty} \hat{\varphi}(k) e_{k}$ with $\hat{\varphi}(k)=0$ for all $k \geqq n_{0}$, where $n_{0}$ is the least positive integer such that $e_{n_{0}} \bar{\varphi}$ is analytic. It then follows from (iii) that $\hat{\varphi}(-k)=0$ for all $k \geqq n_{0}$. Hence $\varphi$ is a trigonometric polynomial. Combining this and Theorem 1 , we can conclude that if $\varphi$ is almost coanalytic, then the Toeplitz operator $T_{\varphi}$ is subnormal if and only if either $T_{\varphi}$ is analytic or normal. This fact immediately suggests the following question: What can one say about the subnormal Toeplitz operator $T_{\varphi}$ if $\varphi$ is almost analytic? We are not able to answer this question.

However, if $T_{\varphi}$ is quasinormal, we can show that $\varphi$ must be a trigonometric polynomial. This is the main result of the following section.

3. Quasinormal Toeplitz operators. The reader is referred to Brown [1] for the definition and elementary facts about quasinormal operators. We first show that the scalar multiples of pure isometries exhaust all the quasinormal analytic Toeplitz operators.

THEOREM 2. For a bounded analytic function $\varphi$, the Toeplitz operator $T_{\varphi}$ is quasinormal if and only if $\varphi$ is a constant multiple of an inner function.

Proof. The sufficiency is again trivial. Conversely, if $T_{\varphi}$ is quasinormal, then $T_{\varphi}$ commutes with $T_{\varphi}^{*} T_{\varphi}$. Since $\varphi$ is analytic, we have $T_{\varphi}^{*} T_{\varphi}=T_{|\varphi|^{2}}$. So $T_{|\varphi|^{2}}$ commutes with $T_{\varphi}$. It follows that $|\varphi|^{2}$ is analytic [2, Theorem 9]. Therefore $|\varphi|$ is a constant function $c$ because $|\varphi|^{2}$ is a real analytic function. Hence $\varphi=c \chi$ and $\chi$ is inner. The proof is complete.

We have seen in Remark 1 that, for almost coanalytic (bounded) functions $\varphi$, we have a relatively easy solution to Halmos' problem. However, for almost analytic $\varphi$, we shall show that quasinormality of $T_{\varphi}$ implies $\varphi$ is a trigonometric polynomial. The proof seems to be somewhat nontrivial.

THEOREM 3. Let $\varphi$ be a bounded almost analytic function. If the Toeplitz operator $T_{\varphi}$ is quasinormal, then either $\varphi$ is a constant multiple of an inner function, or $\varphi$ is a nonanalytic trigonometric polynomial, in which case $T_{\varphi}$ is normal. 
Proof. Assume $\varphi$ is not analytic. Then there is some positive integer $n_{0}$ such that $\varphi(z)=\bar{z}^{n_{0}} h(z)$ where $h$ is analytic and $\hat{h}(0) \neq 0$. It is well known that $h=\chi F$ where $\chi$ is inner and $F$ is outer ${ }^{2}[6]$. Therefore $T_{\varphi}=U^{* n_{0}} T_{\chi} T_{F}$. First we show that $F$ is an analytic polynomial of degree $2 n_{0} . T_{\varphi}\left(H^{2}\right)=$ $U^{* n_{0}} T_{h}\left(H^{2}\right)$. So $\left(T_{\varphi}\left(H^{2}\right)\right)^{-}=\left(U^{* n_{0}}\left(T_{h} H^{2}\right)^{-}\right)^{-}$(the bar means closure this time). Since $\left(T_{h}\left(H^{2}\right)\right)$ is the smallest invariant subspace of $U$ containing $h$, we see that $\left(T_{h}\left(H^{2}\right)\right)=\chi \cdot H^{2}$ [6]. Therefore $U^{* n_{0}}\left(e_{n} \chi\right)$ is in $\left(T_{\varphi}\left(H^{2}\right)\right)$ for all $n \geqq 0$. In particular, $e_{n_{0}} \chi$ is in $\left(T_{\varphi}\left(H^{2}\right)\right)$. Note that $U^{* n_{0}}\left(e_{2 n_{0}} \chi\right)=e_{n_{0}} \chi$. Moreover, since $T_{\varphi} T_{\varphi}^{*}=T_{\varphi}^{*} T_{\varphi}$ on $\left(T_{\varphi}\left(H^{2}\right)\right)$, we have

This means

$$
T_{\varphi} T_{\varphi}^{*}\left(e_{n_{0}} \chi\right)=T_{\varphi}^{*} T_{\varphi}\left(e_{n_{0}} \chi\right)
$$

But

$$
\left\|T_{\varphi}\left(e_{n_{0}} \chi\right)\right\|=\left\|T_{\varphi}^{*}\left(e_{n_{0}} \chi\right)\right\|
$$

$$
\begin{aligned}
\left\|T_{\varphi}\left(e_{n_{0}} \chi\right)\right\| & =\left\|U^{* n_{0}} T_{h}\left(e_{n_{0}} \chi\right)\right\|=\left\|U^{* n_{0}}\left(e_{n_{0}} \chi h\right)\right\| \\
& =\|\chi h\|=\|\chi \chi \cdot F\|=\|F\|=\left\|e_{2 n_{0}} F\right\|, \\
\left\|T_{\varphi *}\left(e_{n_{0}} \chi\right)\right\| & =\left\|T_{h}^{*} U^{n_{0}}\left(e_{n_{0}} \chi\right)\right\|=\left\|T_{h}^{*}\left(e_{2 n_{0}} \chi\right)\right\| \\
& =\left\|P\left(\bar{\chi} F \chi e_{2 n_{0}}\right)\right\|=\left\|P\left(e_{2 n_{0}} F\right)\right\| .
\end{aligned}
$$

Thus $P\left(e_{2 n_{0}} \bar{F}\right)=e_{2 n_{0}} \bar{F}$, so $e_{2 n_{0}} \bar{F}$ is analytic. Therefore, $F$ is an analytic polynomial of degree at most $2 n_{0}$. On the other hand, consider $U^{* n_{0}}\left(e_{2 n_{0}-1} \chi\right)=e_{n_{0}-1} \chi$, which we know is in $\left(T_{\varphi}\left(H^{2}\right)\right)^{-}$, we have

$$
\begin{aligned}
\left\|T_{\varphi}\left(e_{n_{0}-1} \chi\right)\right\|^{2} & =\left\|U^{* n_{0}}\left(e_{n_{0}-1} \chi \cdot h\right)\right\|^{2}=\left\|U^{*}(\chi \cdot h)\right\|^{2} \\
& =\|\chi h\|^{2}-|\hat{\chi}(0) \hat{h}(0)|^{2}=\|F\|^{2}-|\hat{\chi}(0) \hat{h}(0)|^{2} \\
& <\|F\|^{2}=\left\|e_{2 n_{0}-1} F\right\|^{2} .
\end{aligned}
$$

(Note that $\hat{h}(0) \neq 0$ and $\hat{h}(0)=\hat{\chi}(0) \hat{F}(0)$.)

$$
\left\|T_{\varphi}^{*}\left(e_{n_{0}-1} \chi\right)\right\|^{2}=\left\|P\left(h e_{2 n_{0}-1} \chi\right)\right\|^{2}=\left\|P\left(e_{2 n_{0}-1} \bar{F}\right)\right\|^{2} .
$$

Since $\left\|T_{\varphi}^{*}\left(e_{n_{0}-1} \chi\right)\right\|=\left\|T_{\varphi}\left(e_{n_{0}-1} \chi\right)\right\|$, we have

$$
\left\|P\left(e_{2 n_{0}-1} \bar{F}\right)\right\|<\left\|e_{2 n_{0}-1} \bar{F}\right\| .
$$

Therefore $e_{2 n_{0}-1} F$ is not analytic and $F$ has degree at least as large as $2 n_{0}$. Consequently $F$ is an analytic polynomial of degree $2 n_{0}$.

We are ready to prove that $h$ is an analytic polynomial of degree at most $2 n_{0}$.

Since $T_{\varphi} T_{\varphi}^{*} T_{\varphi}=T_{\varphi}^{*} T_{\varphi} T_{\varphi}$, we have

$$
U^{* n_{0}}\left(T_{\varphi} T_{\varphi}^{*} T_{\varphi}\right) U^{n_{0}}=U^{* n_{0}}\left(T_{\varphi}^{*} T_{\varphi} T_{\varphi}\right) U^{n_{0}}
$$

\footnotetext{
${ }^{2}$ An analytic function is said to be outer if it is cyclic for the unilateral shift.
} 
We see that

$$
\begin{aligned}
U^{* n_{0}}\left(T_{\varphi} T_{\varphi}^{*} T_{\varphi}\right) U^{n_{0}} & =U^{* n_{0}}\left(U^{* n_{0}} T_{h}\right)\left(T_{h} U^{n_{0}}\right)\left(U^{* n_{0}} T_{h}\right) U^{n_{0}} \\
& =U^{* 2 n_{0}} T_{h} T_{h} T_{h} U^{n_{0}}=U^{* 2 n_{0}} T_{h} T_{\hbar_{h e_{n_{0}}}}=T_{e_{-2 n_{0}}{ }_{h}} T_{h h_{n_{0}}}
\end{aligned}
$$

and that

$$
U^{* n_{0}}\left(T_{\varphi}^{*} T_{\varphi} T_{\varphi}\right) U^{n_{0}}=T_{e_{-n_{0}} \hbar h h} .
$$

The above computation is based on the multiplicative properties of Toeplitz operators [2, Theorem 9].

Now

$$
\left(T_{e_{-2 n_{0}} h}\right)\left(T_{\hbar h e_{n_{0}}}\right)=T_{e_{-n_{0}} \hbar h h} .
$$

The above equality holds if and only if either $e_{-2 n_{0}} h$ is coanalytic or $\bar{h} h e_{n_{0}}$ is analytic [2, Theorem 8]. But $\bar{h} h e_{n_{0}}=\bar{F} F e_{n_{0}}$, and degree of $F$ is $2 n_{0}$. Therefore $F F e_{n_{0}}$ cannot be analytic. So $e_{-2 n_{0}} h$ is coanalytic. Therefore $h$ is an analytic polynomial of degree at most $2 n_{0}$. Consequently $\varphi=e_{-n_{0}} h$ is a trigonometric polynomial, and it is not analytic because $\hat{h}(0) \neq 0$. Furthermore quasinormality implies subnormality [4, Problem 154]. Therefore by Theorem 1 we can conclude that $T_{\varphi}$ is normal. This completes the proof.

REMARK 2. In the proof of the Theorem 3, we showed that $\varphi=e_{-n_{0}} h$ is a nonanalytic trigonometric polynomial of the form

$$
\varphi=\hat{\varphi}\left(-n_{0}\right) e_{-n_{0}}+\cdots+\hat{\varphi}\left(m_{0}\right) e_{m_{0}}
$$

where $0 \leqq m_{0} \leqq n_{0}, \hat{\varphi}\left(-n_{0}\right)=\hat{h}(0) \neq 0, \hat{\varphi}\left(m_{0}\right) \neq 0$. Since $T_{\varphi}$ is hyponormal, it follows from the inequality (iii) in $\S 2$ that $m_{0} \geqq n_{0}$. Hence $m_{0}=n_{0}$. We rewrite $\varphi=\hat{\varphi}\left(-n_{0}\right) e_{-n_{0}}+\cdots+\hat{\varphi}(0)+\cdots+\hat{\varphi}\left(n_{0}\right) e_{n_{0}}$. The normality $T_{\varphi}$, as we have already shown, follows from Theorem 1 . However, we present here a different proof of this fact which does not depend on Theorem 1. The proof goes like this.

$$
\begin{aligned}
& T_{\varphi}^{*} T_{\varphi} T_{\varphi} e_{2 n_{0}-1}=P\left(\bar{\varphi} \varphi \varphi e_{2 n_{0}-1}\right)-\hat{\varphi}\left(-n_{0}\right)^{2} P\left(e_{-1} \bar{\varphi}\right), \\
& T_{\varphi} T_{\varphi}^{*} T_{\varphi} e_{2 n_{0}-1}=P\left(\varphi \bar{\varphi} \varphi e_{2 n_{0}-1}\right)-\left(\hat{\varphi}\left(n_{0}\right)\right)-\hat{\varphi}\left(-n_{0}\right) P\left(e_{-1} \varphi\right) .
\end{aligned}
$$

The quasinormality of $T_{\varphi}$ implies that

$$
\hat{\varphi}\left(-n_{0}\right)^{2} P\left(\bar{\varphi} e_{-1}\right)=\left(\hat{\varphi}\left(n_{0}\right)\right)-\hat{\varphi}\left(-n_{0}\right) P\left(\varphi e_{-1}\right) .
$$

Comparing the Fourier coefficients on both sides of $(*)$, we have

$$
\hat{\varphi}\left(-n_{0}\right)(\hat{\varphi}(-k))^{-}=\left(\hat{\varphi}\left(n_{0}\right)\right)^{-} \hat{\varphi}(k) \text { for } k=1,2, \cdots, n_{0} \text {. }
$$

Let $\alpha$ be such that $\hat{\varphi}\left(-n_{0}\right)=\alpha\left(\hat{\varphi}\left(n_{0}\right)\right)$. Then $|\alpha|=1$. It follows that $\hat{\varphi}(-k)=\alpha(\hat{\varphi}(k))-$ for $k=1,2, \cdots, n_{0}$. Therefore $T_{\varphi}$ is normal.

It is known that the unilateral shift $U$ is unitarily equivalent to the 
analytic Toeplitz operator $T_{B}$ defined by the inner function $B(z)=$ $(z-\alpha) /(1-\bar{\alpha} z)$ where $|\alpha|<1$ [7, Chapter 7]. This fact leads immediately to the following corollary to Theorem 3 .

CoRollary. Let $\varphi$ be a bounded function of the form $\bar{B}^{n_{0}} h$ for some positive integer $n_{0}$, where $B(z)=(z-\alpha) /(1-\bar{\alpha} z)$ with $|\alpha|<1$, and $h$ is analytic. If the Toeplitz operator $T_{\varphi}$ is quasinormal, then either $\varphi$ is a constant multiple of an inner function or $T_{\varphi}$ is normal.

Proof. Let $V$ be the unitary operator on $H^{2}$ such that $V^{*} T_{B} V=U$. Then $V^{*} T_{\varphi} V=U^{* n_{0}}\left(V^{*} T_{h} V\right)$. On the other hand $U\left(V^{*} T_{h} V\right)=V^{*} T_{B} T_{h} V=$ $V^{*} T_{h} T_{B} V=\left(V^{*} T_{h} V\right) U$. Since $V^{*} T_{h} V$ commutes with $U$, it is an analytic Toeplitz operator [2]. Thus $V^{*} T_{\varphi} V=U^{* n_{0}} T_{h_{1}}$ where $h_{1}$ is the analytic function such that $T_{h_{1}}=V^{*} T_{h} V$. It follows from Theorem 3 that $V^{*} T_{\varphi} V$ is either analytic or normal. If it is normal, so is $T_{\varphi}$. If $V^{*} T_{\varphi} V$ is an analytic Toeplitz operator, then $U\left(V^{*} T_{\varphi} V\right)=\left(V^{*} T_{\varphi} V\right) U$. Hence

$$
V^{*}\left(T_{B} T_{\varphi}\right) V=V^{*}\left(T_{\varphi} T_{B}\right) V
$$

Consequently, $T_{B} T_{\varphi}=T_{\varphi} T_{B}$. But $T_{B}$ is analytic, so $T_{\varphi}$ must be analytic. This follows from the commutativity property of Toeplitz operators [2, Theorem 9]. Hence $\varphi$ is analytic and $T_{\varphi}$ is quasinormal. We conclude that $\varphi$ is a constant multiple of an inner function by Theorem 2. This completes the proof.

REMARK 3. We note that for functions of the form $\bar{B} h$, where $h$ is analytic and $B$ is a Blaschke product (not of a single factor), the argument that proved the corollary does not go through. However if the function $\varphi$ is of the form $\bar{g} F$ where $g$ is analytic and $F$ is outer, then one can easily see that the Toeplitz operator $T_{\varphi}$ has dense range. Hence quasinormality of $T_{\varphi}$ implies normality of $T_{\varphi}$.

REMARK 4. Before closing this paper, we would like to make a few remarks on hyponormal Toeplitz operators. The Toeplitz operator $a U^{* n_{0}}+b U^{n_{0}}$ with $0<|a|<|b|$ and $n_{0}>0$ is seen to be a hyponormal Toeplitz operator. It follows from Theorem 1 that this operator is not subnormal, hence not normal. The nonnormality of this operator can also be easily seen from the fact that $|a| \neq|b|$. In general, let $\varphi$ be a trigonometric polynomial of the form $\varphi=\sum_{k=-n_{0}}^{n_{0}} \hat{\varphi}(k) e_{k}$ where $n_{0}>0$ and $\left|\hat{\varphi}\left(n_{0}\right)\right|=$ $\left|\hat{\varphi}\left(-n_{0}\right)\right| \neq 0$. Then hyponormality of $T_{\varphi}$ implies normality of $T_{\varphi}$. The proof goes like this. The self-commutator $C_{\varphi}=T_{\varphi}^{*} T_{\varphi}-T_{\varphi} T_{\varphi}^{*}$ has range contained in the finite dimensional subspace spanned by $e_{0}, e_{1}, \cdots, e_{n_{0}-1}$. The $i$-jth entry of the matrix of $C_{\varphi}$ (with respect to the usual basis $e_{k} ; k \geqq 0$ ) is given by

$$
\alpha_{i, j}=\sum_{\nu \geqq 0}(\hat{\varphi}(-i+v))^{-} \hat{\varphi}(-j+v)-\sum_{\nu \geqq 0} \hat{\varphi}(i-v)(\hat{\varphi}(j-v))^{-} .
$$


We have $\alpha_{i j}=0$ for all $i \geqq n_{0}$ or $j \geqq n_{0}$. Thus the positive infinite matrix $\left(\alpha_{i j}\right)$ is of the form

$$
\left(\begin{array}{ccc|c}
\alpha_{00} & \cdots & \alpha_{0, n_{0}-1} & 0 \\
\vdots & & \vdots & 0 \\
\alpha_{n_{0}-1,0} & \cdots & \alpha_{n_{0}-1, n_{0}-1} & \\
\hline & 0 & & 0
\end{array}\right)
$$

For $0<k<n_{0}$, the 2-by-2 matrix

is also positive.

$$
A=\left(\begin{array}{ll}
\alpha_{k-1, k-1} & \alpha_{k-1, n_{0}-1} \\
\alpha_{n_{0}-1, k-1} & \alpha_{n_{0}-1, n_{0}-1}
\end{array}\right)
$$

An easy computation shows that

$$
\begin{aligned}
\alpha_{n_{0}-1, n_{0}-1} & =\left|\hat{\varphi}\left(n_{0}\right)\right|^{2}-\left|\hat{\varphi}\left(-n_{0}\right)\right|^{2}=0, \\
\alpha_{k-1, n_{0}-1} & =\left(\hat{\varphi}\left(n_{0}\right)\right)-\hat{\varphi}(k)-(\hat{\varphi}(-k))-\hat{\varphi}\left(-n_{0}\right), \\
\alpha_{n_{0}-1, k-1} & =\left(\alpha_{k-1, n_{0}-1}\right) .
\end{aligned}
$$

The positive definiteness of the matrix $A$ implies that $\alpha_{k-1, n_{0}-1}=0$. Therefore we have $\left(\hat{\varphi}\left(n_{0}\right)\right)^{-} \hat{\varphi}(k)=(\hat{\varphi}(-k))-\hat{\varphi}\left(-n_{0}\right)$ for all $0<k<n_{0}$. Hence $\hat{\varphi}(k)=\alpha(\hat{\varphi}(-k))^{-}$for some $\alpha$ with $|\alpha|=1$. This implies that $T_{\varphi}$ is normal.

\section{REFERENCES}

1. A. Brown, On a class of operators, Proc. Amer. Math. Soc. 4 (1953), 723-728. MR 15, 538.

2. A. Brown and P. R. Halmos, Algebraic properties of Toeplitz operators, J. Reine Angew. Math. 213 (1963/64), 89-102. MR 28 \#3350; errata MR 30, 1205.

3. P. R. Halmos, Ten problems in Hilbert space, Bull. Amer. Math. Soc. 76 (1970), 887-933. MR 42 \#5066.

4. - , A Hilbert space problem book, Van Nostrand, Princeton, N.J., 1967. MR 34 \#8178.

5. - Shifts on Hilbert spaces, J. Reine Angew. Math. 208 (1961), 102-112. MR 27 \#2868.

6. H. Helson, Lectures on invariant subspaces, Academic Press, New York, 1964. MR 30 \#1409.

7. K. Hoffman, Banach spaces of analytic functions, Prentice-Hall Series in Modern Analysis, Prentice-Hall, Englewood Cliffs, N.J., 1962. MR 24 \#A2844.

Department of Mathematics, Wayne State University, Detroit, Michigan 48202 